\title{
Provides AN APPRoACH BASEd on AdAPTIVE FORWARDING AND LABEL SWITCHING TO IMPROVE THE SPEED OF PACKET FORWARDING IN NDN NETWORKS
}

\author{
Hadis Rezaei, Sahar Sadeghi and Leila Badeli \\ Department of Computer Engineering, \\ Science and Research Tehran Branch, Tehran, Iran
}

\begin{abstract}
Named Data Networking (NDN) is a recently designed Internet architecture that benefits data names instead of locations and creates essential changes in the abstraction of network services from "delivering packets to specific destinations" to "retrieving data with special names" makes. This fundamental change creates new opportunities and intellectual challenges in all areas, especially network routing and communication, communication security, and privacy. The focus of this dissertation is on the forwarding aircraft feature introduced by NDN. Communication in NDN is done by exchanging interest and data packets. Consumers send interest packets to request data, routers send them based on the data prefix name, and manufacturers respond with data packets exactly how interest is received. During this process, routers maintain information about the status of suspended interests. As regards NDN is still in its early stage, none of these strong efficiency features have done systematically designed, measured, or tracked in data retrieval and multi-path detection. This paper tries to improve transport performance by combining label switching with adaptive transport methods. For this purpose, in addition to RTT, another timer called $T$ is used, an RTT factor for interface rating. And uses the BDP to choose the best interface to respond to interest. During this process, response time, throughput, and data recovery time are improved by $12 \%$, $10 \%$, and $5 \%$, respectively.
\end{abstract}

\section{KEYWORDS}

NDN, BDP, RTT, CCN, Ranking interfaces, Name label Switching.

\section{INTRODUCTION}

Since the 1970s, the network architecture relying on TCP/IP protocols has faced many increasingly earnest challenges, such as network security, reliability, flexibility, mobility, congestion control, and resource allocation [1]]. According to recent prophecy, global IP traffic will provide a peaking approach in the coming years. Most of the traffic is due to various types of video, Internet that will account for almost 90\% of global consumer traffic by 2015[2]. Communication by distributed content proves the ineffectiveness of the existing architecture, which led to the development of data-centric networks or the Information-Centric Networking (ICN) $[3,4,5,6,7]$. Communication with distributed content reflects the lack of existing implicit architecture, which led to the development of data-centric networks or the ICN. Therefore, many variants of the ICN are worked out to affect the need of the future network. There are various types of content-centric networking [8]. The last architecture defined for content-centric networks is named data networking (NDN) $[9,10]$. 
Named Data Networking (NDN) [11, 12] is the noted design of newly proposed ICN architectures. It displaces the hourglass figure of the IP architecture but changes the end-to-end data rendition model at the slim middle by a receiver-driven data retrieval sample. Consequently, NDN alteration the network service semantics via "deliver packets to given addresses" to "retrieving data of given names." NDN packets convey data names rather than source and goal addresses $[13,14]$. Every connection in NDN is performed using two different types of packets: Interest and Data. Both packets transport a name that uniquely recognizes a part of data that can be taken away in one packet. The name of the data is structured in the NDN hierarchy; similar to IP packet transfer, an NDN network accomplishes the best attempt for data retrieval. An Interest or Data packet can be missing. It is the ending consumer's accountability to retransmit the interest if it does not accept the favorite Data then attend round trip time (RTT), and it still needs the data [15].

NDN forwarding process is divided into a two-step interest packet sent by consumers and returned along the same path in reverse. The NDN router forwarding packet by PIT table leads consumers. The process forwarding enables the NDN router; it will replace other paths when the link failure occurs. This feature is called Adaptive forwarding in NDN [16] Due to datagram NDN attendant cost associated. Moreover, using the RTT to rank the interface in the FIB is a long time.

Moreover, when the network grows, the FIB size increases and the FIB entry stored in the FIB entry will be ineffective in searching for the name. So scalable structure at the FIB table facilitates the search for the title and short forwarding packets. The exchange of packets in the NDN is based on FIB information. Unlike the routing table in IP-based networks that have fixed address lengths, The FIB table in the NDN has a name prefix with variable length. In addition, a plurality of references will be faced with excessive growth PIT table, NDN is the first network architecture to prepare an adaptive forwarding plane. With adaptive forwarding, NDN routers can indicate and retrieve from network problems quickly without waiting for universal routing looking for. For example, NDN routers can approximate the round-trip time (RTT) for each name prefix per confiscation within Interests and attentive Data packets coming back. Network problems can be detected by setting up a timer relaying on the RTT estimation. If data is not coming before the timer expires, there may be possible forwarding problems in the network. Once issues are discovered, NDN routers can start tracking other paths directly using local state information. Since Interest forwarding is loop-free, routers can efficiently operate in several ways to improve from the problems. The plan to achieve the goals uses the scheduler RTT, Interest NACK, Interface ranking [15].

\section{OVERVIEW OF NDNS NETWORK ForWARDING}

NDN architecture is like to IP but only in the hourglass thin waist, the section that fundamental changes have happened in this architecture via replacing interest name with IP address. Entire communication in NDN is taken by two types of packets that are called interest and data packets. Similar to IP packet transition, NDN network forwarding has the best effort in data detection but during this process might an Interest or Data packet can be lost. if the consumer does not receive the desirable Data after awaiting round trip time (RTT) and still wants the data regarding the NDN datagram consumer can send the Nack interest to the downstream routers that stop their transmission. However, inverse IP architecture uses the destination address to forward requests, NDN structure uses the name of data to satisfy consumer requests. This primary difference in a plan leads to two profound differences in data delivery operations that are in the CS and security [12]. 
Every NDN router contains three basic data structures: A Content Store (CS) for storing received data packets temporarily, a Pending Interest Table (PIT), and a forwarding table (FIB) (see Fig. 1). Via its name, each PIT entry storage and Interest packet has been consigned, looking for the Data packet to relapse. The entry storage of the name, the incoming and outgoing interface of the interest, has been forwarded to. An NDN router's FIB is carelessly like the FIB in an IP router barring then it contains name prefixes instead of IP address prefixes, and it can display many interfaces for a given name prefix. Every NDN router has a strategic measure that makes forwarding decisions for every Interest packet. When a router takes an Interest packet, it first checks if there is an according to data in its CS [16]. If a match is found therefore the data is returned to the incoming interface of the Interest packet. Whether not, the Interest name is perused to the entries in the PIT. If the name was in the PIT formerly, it can be either a repetitive Interest that should be released or interest retransferred by the consumer. Can necessity be forwarded using a several, outgoing interface, or an Interest from other consumers requesting similar data that needs the incoming interface of this interest to existing added to the available PIT entry? If the name does not stand in the PIT, interest is increased and forwarded to the interface selected by the strategy module. When a Data packet is accepted, its name is used to regard the PIT. If an according PIT entry is found, the router sends the data packet into the interface at the interest received, stores the data in the Content Store, and pickups the PIT entry. Differently, the Data packet is rejected. Each interest also has an affiliated life history; the PIT entry is deleted when the life history expires [15].

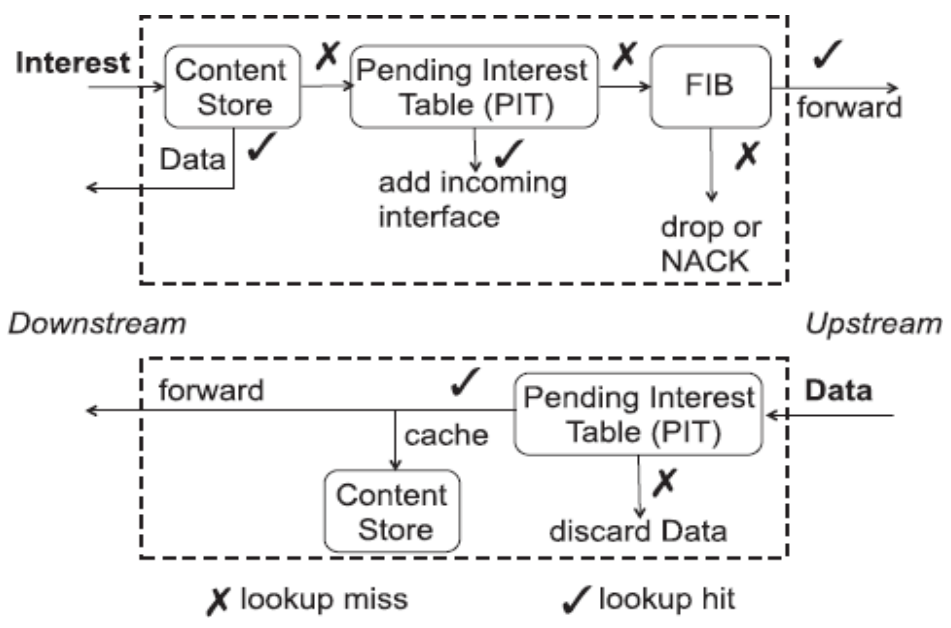

Figure 1. Interest and Data processing in NDN [15]

The NLS forwarding process uses the MPLS mechanism and uses the benefits of labeling packages to reduce the total time faster than name lookup in NDN. According to the destination node labels of incoming packets, the MPLS network is assigned a label edge router (LER) has been called. Then the package forwarding is closed without the need for self-testing, only in the contents of this label. To assign labels, Forwarding Equivalence Classes (FECs) are divided into which are mapped to different Label Switched Paths (LSPs) in the MPLS core. The advantages of the label switching mechanism of MPLS can be explored in forwarding NDN. The NLS domain connects to the NDN network and begins the forwarding process.

\section{Methodology}

The proposed method in this study is combined with three steps, 1) Interface coloring 2), Interfaces ranking, 3) Selecting the best interface and forwarding interest packet. In the following paragraph, each step will describe in detail. 


\subsection{Colouring Interface in FIB}

The first section of the method in this paper as illustrated in Fig (2) is including determining the color for each interface using the two parameters $\mathrm{T}$ and $\mathrm{T} 1$ that consequences, $\mathrm{T} 1$ is equivalent to RTT and T is a factor of RTT. When a new entry in FIB is created or a new entry is added to the FIB entry. The state for all interfaces is yellow. Regarding this point, the state of each interface and their ranking is determined by the RTT scheduler, when the interface is dark green means the response time is smaller than the T1 there for data retrieval is done in the sort time with the high speed. The interface with the light green means in contrast ot the erofeb state the rate of data flow is slow because the response time is smaller than the $\mathrm{T}$ scheduler. A green interface convert to yellow when the interest doesn't satisfy in a certain time or the data flow stops for a certain period. In this respect, when there is no data stream, the interface is red. The dork-green interface is always preferred to the light green interface.

On the other hand, when there is no green interface, yellow is the priority and the red interface is never used for data transfer. Entrance FIB contains the estimate of the RTT in each interface for retrieving stored data which is taken at any time in the received interface of a packet [15]. In order to indicate the status of each interface is used a four-color.

- Light - Green: The interface can retrieve data in a short time.

- Dork -Green: The interface can retrieve the data but the time of retrieval is slower than the light green

- yellow: it is unknown whether the interface can retrieve data or not

- Red: The interface cannot data retrieve

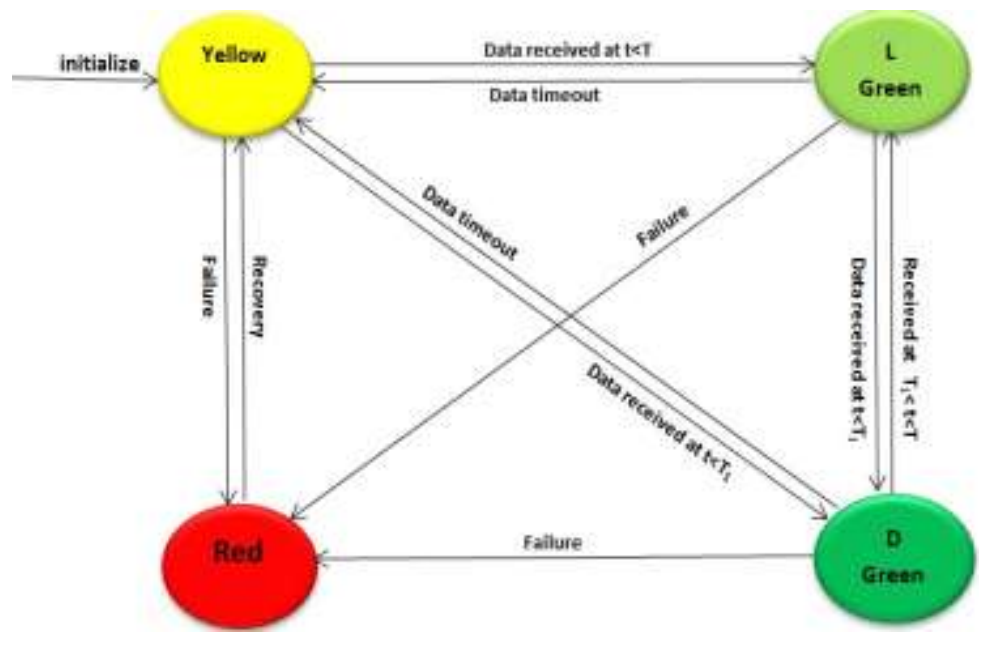

Figure 2. Interface Colour Transition

\subsection{Interfaces ranking in the FIB}

In this section of the proposed method, the interfaces in the FIB incoming ranked in order to select the best interface in forwarding strategy. When a router receives a new prefix name, for this prefix allocated a proper interface based on routing priority. There is a wide spectrum of forwarding policies in the NDN architecture due to the FIB table, e,g, if a policy is FIFO, an interface with a smaller PTT will be selected. 
This paper considered ranking policy is using a parameter called BDP and is the result of the RTT * Bandwidth. Using that we can select the best interface to forward of interest. In this approach among all interfaces that are available, the interface will have opted that has small BDP and with using the bandwidth ranked the yellow interfaces

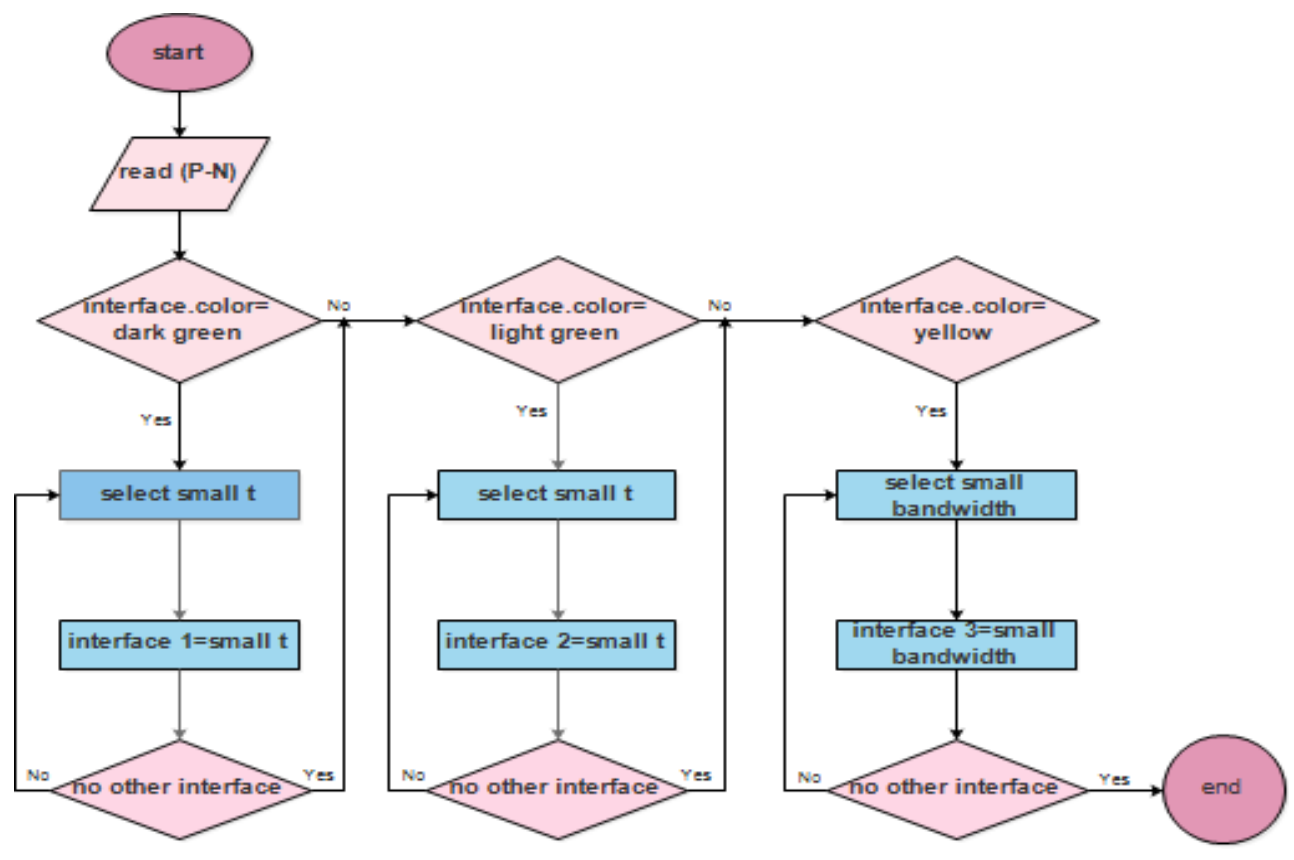

Figure 3. Interfaces ranking in the FIB table

\subsection{Forwarding strategy in the proposed method}

Forwarding strategy in the proposed method as is shown in the Fig (4) has three steps, in this respect, the name that is sent by the consumer will be labeled and would be sent by interest packet. The first step of forwarding is the coloring of the interface which colors for all interfaces are yellow and based on two response times $\mathrm{T}$ and $\mathrm{T} 1$ would be changed to the different colors, e.g. dark green, light green, and red. if the label name requested by the consumer is in the CS, therefore, it transmits to the consumer, otherwise, the PIT table will be reviewed, if this label is not duplicated, the new entry will be created in the PIT entrance and the labeled interest is forwarded to the FIB table. In this, step ranking interface will be done for each desired label, and according to the BDP policy, the interface can be selected that has two properties, firstly be in top priority and has not been used with other requests. Afterward, the PTT scheduler will be started in PIT outcoming, if in $\mathrm{T}$ period of the time, the interest has not responded then the other active interface will be selected, if there was no active interface, Nack interest sent as response and the process of forwarding will be terminated 


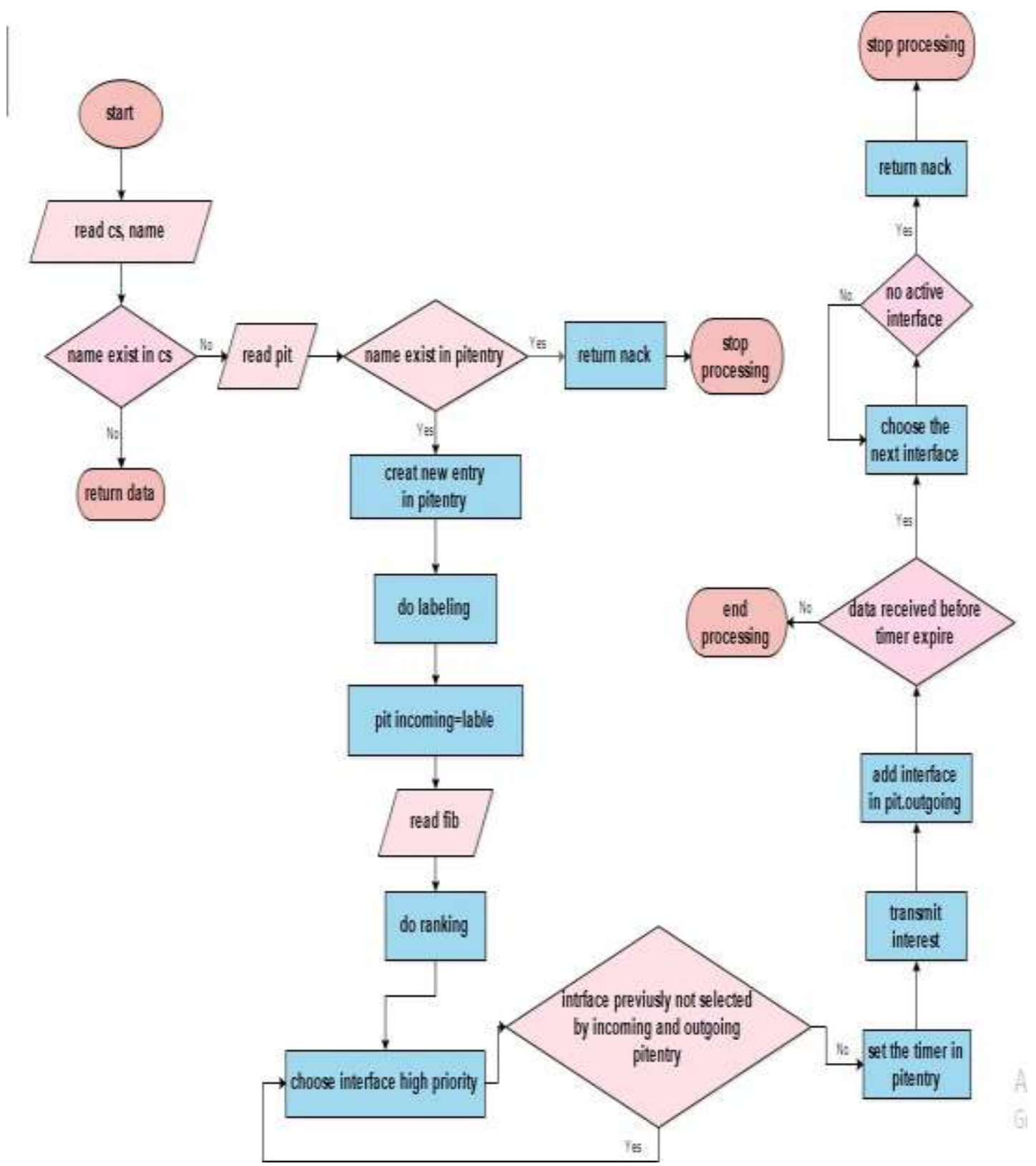

Figure 4. Forwarding strategy in the proposed method

\section{Evaluation}

This paper simulator is used to simulate ndnSIM 0.2 (NDN-based simulator). In this example, ns3 modules are implemented in an NDN communication model. NdnSIM is a common simulation framework designed to examine system features. In this section, the simulation results are used using performance criteria and response time and data retrieval time.

\subsection{Throughput}

Throughput means the amount of total useful processing performed by a computer system over a specified period of time. Or the amount of data that can be exchanged on the network over a period of time. Throughput is usually measured in bits per second and sometimes data packets 
per second or data packets at intervals. The $\mathrm{X}=\mathrm{C} / \mathrm{T}$ equation calculates how to obtain network throughput the that where $\mathrm{X}$ denoted the throughput and $\mathrm{C}$ denoted the number of requests completed by the system. T indicates the total time at which the system was checked.

Figure (5) shows that the network efficiency in the proposed method has increased compared to other methods due to the relevant mechanisms such as ranking the interfaces and selecting the best port for sending data, as well as the simulation time. The rate of useful data traffic has also increased. The AF method, which is the basic method in this paper, has also had a useful performance due to the use of adaptive forwarding. The NLSR [17] method, which in addition to using the similar labeling method of MPLS networks also uses strategies in NDN thus, has shown relatively good performance; But the IP network, which is the main difference between these three models in the presence of CS, is certainly less efficient than these three methods.

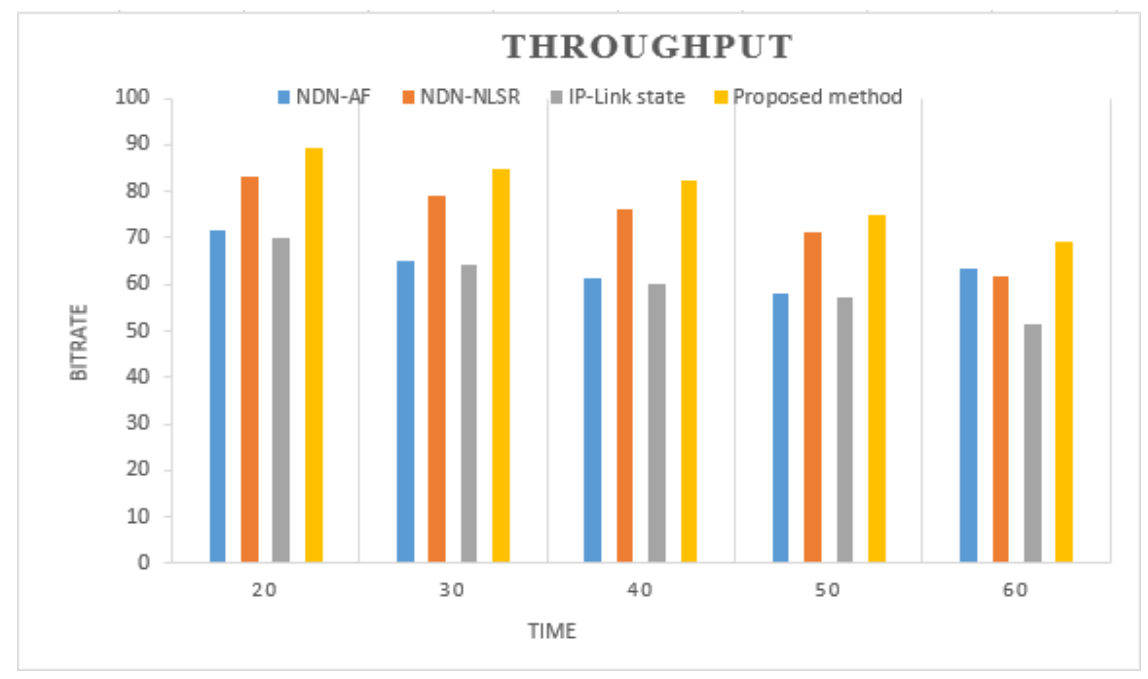

Figure 5. Comparison of throughput

\subsection{Response Time}

The time between sending a request and receiving a response between the source and destination nodes is called the response time, which is measured in milliseconds. The simulation results show that the proposed algorithm performs better at different times. According to Fig (6), the response time usually increases with the number of routers. Due to the applications of adaptive forwarding in data transmission, as well as the labeling of interest packages and looking for data in the shortest part of the prefix name ( $\mathrm{T}$ safix), the response time of the proposed algorithm was less than other methods, but at the beginning of the simulation because there is no knowledge of the state of routers, the interfaces are considered yellow and the sending strategy used will be flooded. Thus, at the moment of starting the desired package, all the interfaces in FIB are sent and it waits from which interface the answer will reach us sooner until using the same gateway for subsequent similar requests. Each node controls the performance of its interface, and if it drops below a certain level, it re-enters the search phase and finds the appropriate interface. This strategy does not use interface ranking and coloring. Therefore, at the beginning of the simulation, the response time was higher than the number of routers, and on average, the response time improved compared to the adaptive forwarding method and other methods._In the case of the NLSR method, which uses the RTT timer and hierarchical naming to send interest, the response time is improved and roughly equal to the base article(AF). But the IP method, whose sending and delivery policies are different from the NDN method, so the response time is much 
longer than the methods mentioned; And as the simulation time increases in proportion to the number of routers, the response time will be higher.

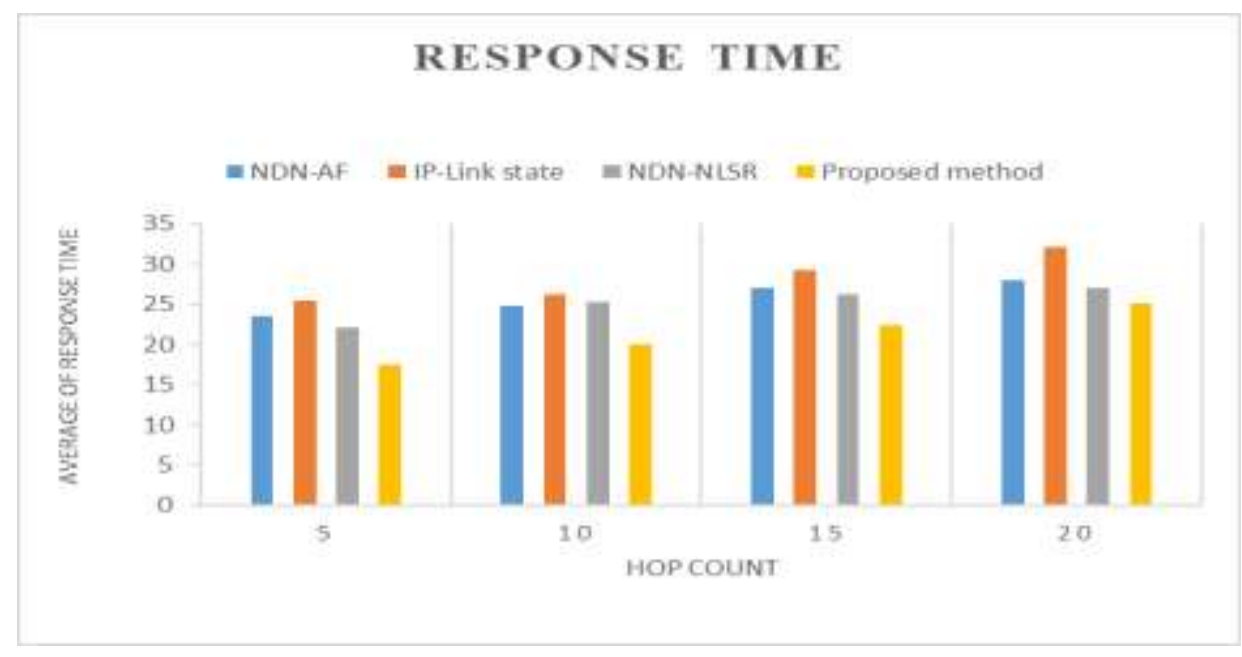

Figure 6. Comparison of response time

\subsection{Processing Time (Delay)}

A packet transmitted a set of routers on its way between the source and the destination system. In exchange for moving a packet between two nodes in the network (host or router), different types of latency are imposed on the transit packet, which affects the total time elapsed between the source and destination of the packet. Bandwidth is just one of the factors that affect the speed of a network. Delay, which indicates the amount of data processing delay in the network, is another important element in evaluating the performance and speed of a network that is closely related to bandwidth. In theory, the bandwidth ceiling is fixed, the actual bandwidth is variable and can cause a delay in a network. Excessive delays in data processing in the network and in a short period of time can cause a crisis in the network and the consequence is to prevent data movement on the transmission medium and reduce bandwidth. According to the results seen in Fig (7), the processing time is better than the other two methods due to the appropriate sending strategy in the proposed method and the basic method (AF), but the proposed method due to the use of two timers and longer calculation time for selecting the appropriate interface in each router to send data has caused latency in the network. In this scenario, the bandwidth is variable and the number of requests and the number of nodes is variable compared to the initial simulation time to investigate the network behavior with increasing routing length. Also, the destination node is not specified in the scenario and is considered a variable at different times. Due to the network conditions, the processing time on the routers due to the use of four colors can cause more delay in the network due to the fact that the destination node is changed each time. But the NLSR method, which completely uses the NDN datagram, has been able to perform well due to its capabilities and fragmentation of this framework. And IP is delayed due to not using the appropriate strategy in sending data, especially with increasing time and number of network nodes, and as shown in the simulation diagram, the processing time for IP will be higher. 


\section{PROCESSING TIME}

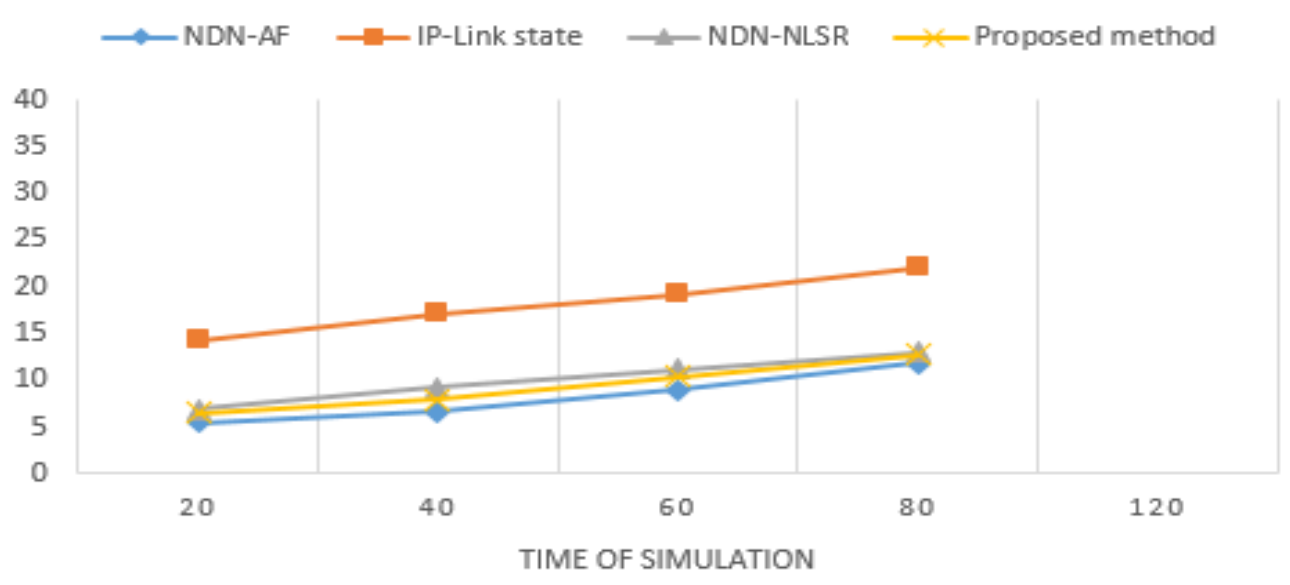

Figure 7. Comparison of Delay

\subsection{Link failure}

Link failure is very common in computer networks. In an ideal circuit-switched network, each packet is delivered to the destination with a percentage of the probability of failure, etc., until the destination is available. The current Internet architecture in an IP-based routing scheme is not responsible for link failure. So that package delivery after failure is not guaranteed, and routing protocols also need convergence time. In the adaptive forwarding scheme, the NDN router is able to manage link failure without routing convergence. In this regard, when link failure is detected by routing protocols, the router will mark the unusable interface; And then it will send the interest to the next interface that has a higher rank. Packets previously sent to the defeated link may still be lost, in which case consumers may be redirected by the router. A router may not have any resu interface to convey interest due to link failure. In such cases, the submission of interests is reduced and consumers will need to resubmit requests after a period of time in order to resume package delivery, which will certainly be a slow and inefficient process. Nack's interest approach has been used to address this issue in comparative guidance. That way, if a router has no way to satisfy an interest, Nack will send a message to the downstream router to find alternative routes if possible, without waiting for it to be sent again.

What has been considered in this experiment is the percentage of data recovery after link failure and the average time of data recovery in AF, NLSR, and proposed methods and alternative route selection. In the designed scenario, after the failure, the selected path is longer than the original path, and it is obvious that the data recovery time increases significantly compared to the nonfailure state. In this designed scenario, the probability of link failure is considered $10 \%$; And using the cumulative distribution function (CDF) in 1000 scenarios link failure has occurred. And the recovery time includes a request that was sent on the network and did not receive a response, and this request was resent by the consumer. What has considered in this experiment is the percentage of data recovery after link failure and the average time of data recovery in AF, NLSR, and proposed methods and alternative route selection. In the designed scenario, after the failure, the selected path is longer than the original path, and it is obvious that the data recovery time increases significantly compared to the non-failure state. In this designed scenario, the probability of link failure is considered 10\%; And using the cumulative distribution function (CDF) in 1000 scenarios link failure has occurred. And the recovery time includes a request that was sent on the network and did not receive a response, and this request was resent by the consumer. In this scenario, packages are considered for which the link failure has occurred. 
According to Figure (8), the results deniatbo erew from the simulation output. In most cases of the proposed method, this time was close to the adaptive guidance method and acted almost similarly. What seems to be important in link breakage is the existence of a Nack in the adaptive forwarding method, which sends this message to downstream routers to control the sending of interest, and downstream routers notice link failure and non-response of the router and another route will choose. However, considering that the proposed method uses two timers, T and T1, and the interfaces are ranked with theirs. The $\mathrm{T}$ parameter is to indicate the state of congestion in the network, which means that in case of high traffic and congestion, a response may be received, but later. Assuming that T1 is smaller than T, T1 is for low-traffic communication and route, and the corresponding interface is dark green, and $\mathrm{T}$ is for the high-traffic route, and the corresponding interface light green. When the interface is dark green, it means that there is no congestion in the network. But if the opposite is true, it indicates that there is a possibility of congestion on the network, and this congestion is the prelude to link failure, and given the application of this strategy and the fact that the BDP parameter is used in the ranking, which is bandwidth and It also takes into account latency, so the smaller the interface with dark green, the greater the likelihood of congestion on the network, and routers will realize this sooner and reduce congestion by controlling packets sent and sending restrictions on packets.

And for this reason, the strategy used will reduce the recovery time to some extent. Meanwhile, NLSR networks that do not use adaptive forwarding fields and only use packet tagging, cannot have a useful function against link failure and packet loss, as shown in Figure (7) The percentage of data recovery in the scenario of this method will be much higher than the other two methods that have been compared. Rather, it is to indicate the state of the network that is congested. If the network is not congested, an answer will be received sooner.

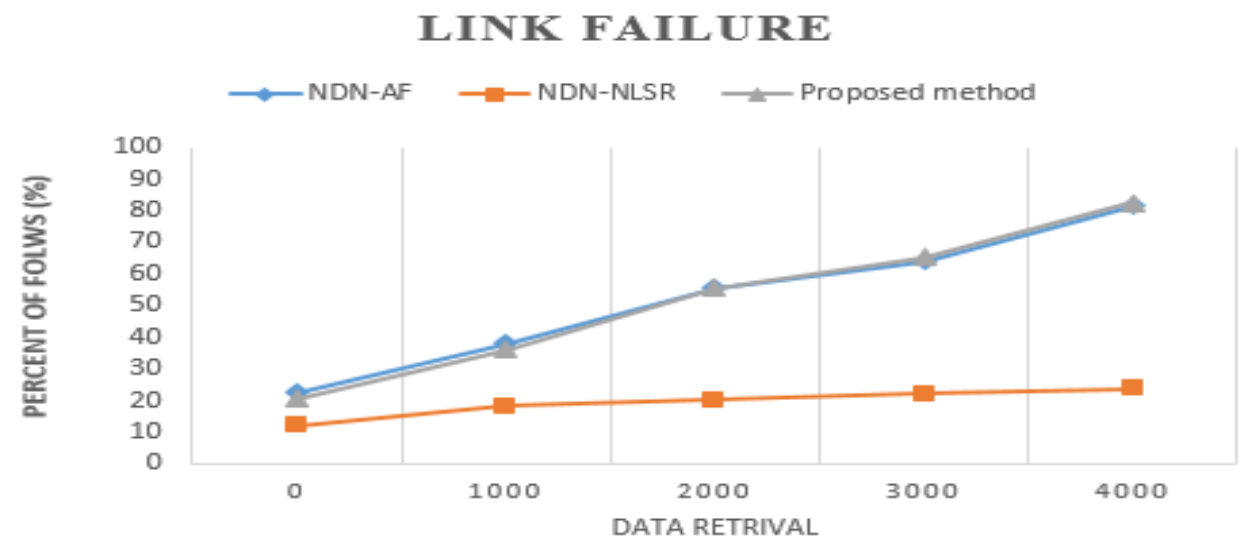

Figure 8. Comparison of link failure

\section{Conclusions}

The current failure of the Internet in the face of various conditions, the main motive and reason for the introduction of data-driven networks that these networks have features such as storage and its signature is an acceptable solution for users and network administrators.

The proposed algorithm is driven to an acceptable level to improve the strategy, using the benefits of adaptive transport and packet labeling. 
By introducing the BDP rating policy and letter labeling, response time and efficiency have improved with comparative transfer and NLS. At the same time, data recovery with the help of a T-timer is improved by comparative adaptive transport.

Since an essential thing in NDN is content and is accessible by name, naming is one of the influential parameters to affect the communication of routers, table size, network time complexity, and many other issues.

Using the naming component encryption method to efficiently encrypt memory for name components and using an array to match the longest prefix name and a comparison guide that uses ranking rankings to send can be an effective way. To speed up the data recovery process. In NDN

\section{REFERENCES}

[1] Moors, T. A critical review of" end-to-end arguments in system design." In Communications, 2002. ICC 2002. IEEE International Conference on. 2002. IEEE.

[2] Dannewitz, C., et al., Network of Information (NetInf)-An information-centric networking architecture. Computer Communications, 2013. 36(7): p. 721-735.

[3] Fotiou, N., D. Trossen, and G.C. Polyzos, illustrating a publish-subscribe internet architecture. Telecommunication Systems, 2012. 51(4): p. 233-245.

[4] Wu, F., Yang, W., Ren, J., Lyu, F., Yang, P., Zhang, Y., \& Shen, X. (2020). NDN-MMRA: Multistage multicast rate adaptation in named data networking WLAN. IEEE Transactions on Multimedia

[5] Abane, A., Muhlethaler, P., \& Bouzefrane, S. (2021). Modeling and improving named data networking over IEEE 802.15. 4. Annals of Telecommunications, 1-12

[6] Lee, Heejin, and Huhnkuk Lim. "Issues on Infotainment Application in Vehicular NDN." Journal of the Korea Institute of Information and Communication Engineering 25, no. 7 (2021): 993-999

[7] Ariefianto, W. T., \& Syambas, N. R. (2017, October). Routing in NDN network: A survey and future perspectives. In 2017 11th International Conference on Telecommunication Systems Services and Applications (TSSA) (pp. 1-6). IEEE.

[8] Jacobson, V., et al. Networking named content. In Proceedings of the 5th international conference on Emerging networking experiments and technologies. 2009. ACM.

[9] Koponen, T., et al. A data-oriented (and beyond) network architecture. in ACM SIGCOMM Computer Communication Review. 2007. ACM.

[10] Zhang, L. et al., Named data networking. ACM SIGCOMM Computer Communication Review, 2014. 44(3): p. 66-73.

[11] Zhang, L., et al., Named data networking (ndn) project. Relatório Técnico NDN-0001, Xerox Palo Alto Research Center-PARC, 2010.

[12] Rezaei, H., \& Vakili, A. (2017). Named data networking: Investigate new infrastructure for ehtfuture of ehtInternet. International Journal of Computer Network and Information Security, 9(1), 40.

[13] Yi, C., et al., Adaptive forwarding in named data networking. ACM SIGCOMM computer communication review, 2012. 42(3): p. 62-67.

[14] Luo, J., et al., Name Label Switching Paradigm for Named Data Networking. Communications Letters, IEEE, 2015. 19(3): p. 335-338.

[15] Yi, C., "ADAPTIVE FORWARDING IN NAMED DATA NETWORKING," 2014. Doctor of Philosophy in the Graduate College the University of Arizona

[16] Yi, C., et al., A case for stateful forwarding plane. Computer Communications, 2013. 36(7): p. 779791.

[17] Wang, L., Lehman, V., Hoque, A. M., Zhang, B., Yu, Y., \& Zhang, L. (2018). A secure link state routing protocol for NDN. IEEE Access, 6, 10470-10482 


\section{AUTHORS}

Hadis Rezaei. She received her bachelor's degree in software engineering from the Islamic Azad University of Saveh, Department of Electrical and Computer, IRAN, in 2012. Later on, she came to Tehran Research Science Branch for Ms. Degree in software engineering in 2013 on Named Data Networking and Content-Centric Networking. She graduated in 2016 with a master's degree.

Sahar Sadeghi. She received her B.SC degree in software engineering from the Islamic Azad University of Saveh department of electrical and computer. Iran, in 2012, she commenced her M.Sc degree in software engineering at the Science and Research Branch University of Tehran, 2013. Her thesis has been on an appropriate filter to detect prostate cancer with increased speed on ultrasound images. She graduated in 2016 with a master's degree.

Lila Badeli. She received her bachelor's degree in IT engineering from Payam nour of Saveh department of Electrical and computer, Iran, in 2011. She graduated from the Science and Research Branch University of Tehran Ms degree in software engineering in 2017 on named Image processing. 